\title{
A Pilot Study to Gather and Evaluate Data on Chiropractic Treatment of LBP and NP
}

\author{
${ }^{1}$ Sudhanva V. Char, Ph.D. \\ ${ }^{2}$ Ralph Davis, DC \\ ${ }^{I}$ AdjunctProfessor, Biostatistics,Department of Nutrition and Dietetics, Life University \\ ${ }^{2}$ Dean, College of Chiropractic, Life University,Marietta, GA 30060
}

\begin{abstract}
:
Objectives:This is a retrospectivenonexperimental observational study with a dual purpose: first,to gather and evaluate pre-and post-treatment statistics at a chiropractic university outpatient teachingclinic using customary instruments of pain and functionality measurement of Low Back Pain $(L B P)$ viz., the Revised Oswestry Disability Index (RODI) and the Neck Disability Index (NDI), the latter for associated Neck Pain (NP). The second purpose is to look into the effectivenessof treatment for indications of LBP and NP.

Methods: The study analyzed patient reported outcomes (PRO) data abouttreatments that patients go through and theresulting improvement in RODI and NDI scores. The pre and post data were then analyzed.

Results:The analysis showedeffectiveness in terms of improvements in functionality as quantified by decreases in RODIand NDI points. The global rate of effectiveness for all patients was computed. Repeated measures or paired samples student $t$ tests done separately on RODI and NDI data indicated significant improvements in both LBP and NP ( $p$-value $=0.0000)$. Evidence did not support linear correlation between age and baseRODI $(r=0.27, p$-value $=0.08042)$. For the subgroup with associated NP linear correlation between RODI and NDI (both before and after treatment) was significant ( $p$-value $=0.0000)$. Incremental improvements in RODI and NDI scores tended to taper off after an optimal number of doses or treatments.

Conclusions:The study substantiated improvements in functionality and reduction in pain following chiropractic intervention. It did not demonstrate significant associations between a) age and baseRODI, and b) incremental improvements in RODI or NDI and treatments (doses) beyond a threshold.
\end{abstract}

Keywords:Effectiveness; Functionality; Patient Reported Outcomes; Evidence Based Research (EBR).

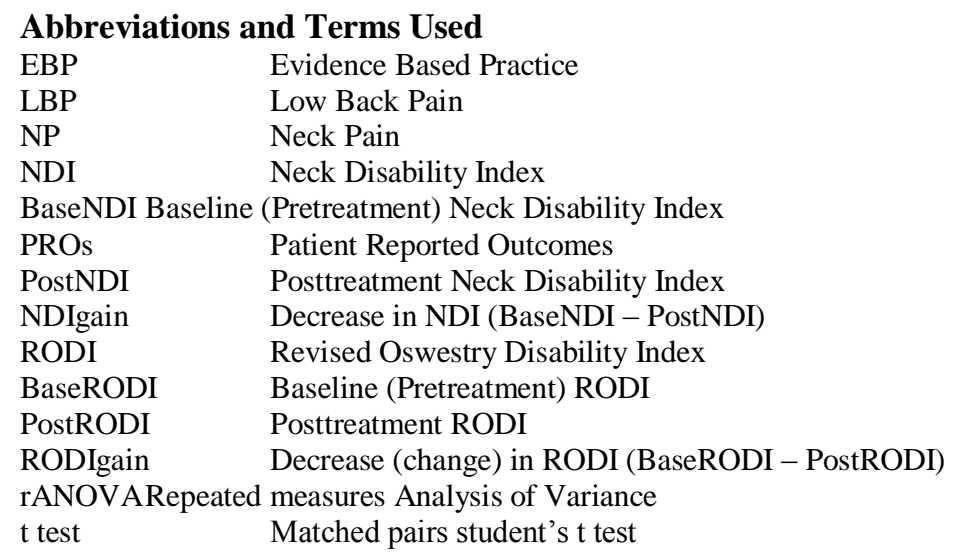

\section{Introduction And Terms Used}

Data recorded conventionally in student teaching clinics help track patient progress and evaluation of chiropractic students. There are diverse other uses for such data such as revealing summary outcomes of treatment for low back pain (LBP) and associated neck pain(NP)somewhat as a comorbidity. Analyses of patient data offer insights into characteristics of the measurement variables. It helps find patterns such as correlations if any, between a) baseline Revised Oswestry Disability Index (BaseRODI) and post-treatment RODI (PostRODI), b) age and baseRODI, c) BaseRODI and baseline neck disability index (NDI), d) number of treatments (doses) and incremental reduction in BaseRODI, e) age and intensity of LBP or Neck Problem (NP) complaints and several others.

Reduction in RODI (BaseRODI minus PostRODI) was termed RODIgain and reduction in NDI (BaseNDI minusPostNDI) was termed NDIgain. 


\section{Efficacy and Effectiveness}

The data for this study comes entirely from patient reported outcomes (PROs) as recorded in the clinic patient files generally, but not exclusively, in response to questions on the revised Oswestry disability index (RODI) form. The baseline data as well as post-treatment data came from the same subject or patient. This is the basis on which the term post hoc data has been used in this study. The term 'pilot study' connotes that no statistical analysis was applied to the clinic patient data prior to this research. There was neither a priori design nor expectations about overall treatment outcomes.

Besides baseline and post hoc data in their respective patient files together withon-line patient demographic profiles constitute the main inputs for this study. The characteristics based on standardized measures such as RODI and NDI, which are in fact structured patient reported outcomes (PROs) are common enough to be generalized to other university chiropractic clinics wherever LBP and NRP are treated. This study is also concerned with effectiveness of interventions in real-world conditions rather than efficacy of the same in optimalor controlled conditions. Efficacy-effectiveness distinctions arearticulatedby Flay ${ }^{[1]}$ et al, Revicki ${ }^{[2]}$ and Frank ${ }^{[3]}$ and others.

\section{Nature of this post hoc study}

An early studyusing patient data to draw inferences about effectiveness of chiropractic treatment for LBP was by Meade et al concluding that "patients receiving chiropractic treatment reported pain and disability scores that were lower than those of the conventionally treated group by a clinically significant amount." ${ }^{[4]}$ This study is more about statistical significance, stating that a particular statistical outcome was not by chance nor is it a false conclusion, and not about being significant enough to alter clinical practice. In a study based on PROs, Nyiendo ${ }^{[5]}$ et al stated that patient satisfaction with chiropractic care for low back pain was significantly higher than traditional medicine.ACC RAC Conference proceedings report retrospective and prospective cases based on PROs. ${ }^{[6]}$ Subjective self-reported outcome measures such as the analog pain sensitivity index (PSI), Roland Morris Questionnaire, the Oswestry Index, the Neck Disability Index, SF-36, SF-12 and others elicit information about patients' disabilities and pains at the time of the first visit to record baseline data and on subsequent visits and treatments to get hold of post-treatment (post hoc) patient data. This study is one such using PROs and post hoc data. ${ }^{[7]}$

Post-hoc or series-based datasetsare a simple and an inexpensive process for gathering basic hypothesis-generating data that subsequently can pave the way for formal, extensive, and fact-finding studies as in the medical field ${ }^{[8]}$ Evidence-based or practice-based research using PROs is instructive in determining which treatments work best for which patients. ${ }^{[9-13]}$ Not the least important is the growing influence of Cochrane rationalization reviews for instituting stringent standards for effectiveness in treatments for health care. The ongoing efforts in the chiropractic field to rationalize intervention procedures for back pain and improving health careare documented by Walker et al and others. ${ }^{[14-18]}$

\section{Effectiveness analysis}

Chiropractic hospital-based intervention research outcomes (CHIRO) studies report on post hoc results and several haveconcluded there were improved outcomes when chiropractic manipulation was included in the treatment. ${ }^{\left[19,{ }^{20]}\right.}$ There was one study for use of special equipment, a Jilco chair protocol for LBPreporting effective outcomes ${ }^{[21]}$ Somewhat similar to evidence-based practice, complete with diagnosis, prognosis and interventions is the work of the Australian Acute Musculoskeletal Pain Guidelines Group. ${ }^{[22}$ Patient reported outcomes (PROs) could serve as a data source if they are routinely and accurately collected and compiled. ${ }^{[23]}$ Augmenting available evidence, like this paper aims to do, would help. Cooper et al state ".... establishing its effectiveness is the hallmark of validating chiropractic as it now exists.",[24]

\section{Methods}

Theobjectives of this research,as noted under the abstract, are to a) gather relevant patient data about LBP and NP from clinic files, b)apply statistical techniques to such data to obtain a summary profile of patients as well asan opinion about effectiveness of treatment for LBP and associated NP, and c) verify associations if any, between variables that may help discern policy-relevant new facts.

The Internal Review Board (IRB) approved use of patient data from files in archives and in active status vide IRB imprimatur dated 10/05/2012.

\section{Selection criteria}

Sample data was collected for 86 patients, 43 male and 43 female, with not much concern for the power of the test such as 50 or 90 percent and the shape of the power curve. The data was given and had a fait accompli status. Nor was there a target effect size the study aimed at: how many visits or doses would be needed to reduce RODI to any desired level. Computations show that for a 0.95 confidence level with a 3 percent margin of error 
when the proportion is not known, the sample size needs to be around 80 . Thus the RODI sample of 86 patients of which the NDI sample of $n=42$ is has enough empirical evidence to yield statistically significant results.

All patients in the sample had LBP as the main health complaint. A subset of 42 patientsalso hadthe comorbidity of NP and in their case the NDI data, both pre and post, was collected and tabulated along with RODI data. NDI data has been used in this study to a relatively lesser extent than RODI, LBP being the primary health complaint, and NP being secondary. Reductions inRODI (RODIgain) and in NDI (NDIgain) are indicators of lessening of pain and improvementsin functionality. The variable 'visits' corresponds to 'treatment' or 'adjustment' or 'dosage'and these four terms are used interchangeably.

Patient files were hand-selectedat random or blind from the clinic shelves and then the patient names were corroborated with an alphabetical patient list with LBP issues made available by the clinic office together with on-line demographic nominal variables such as age and gender. No random generators were used. The required data such as BaseRODI and PostRODI, as well as BaseNDI and PostNDI were collected and tabulated after trying out PSI data and finding RODI more exact. Patients' RODI and NDI were measured several times at different points of time on the same patients before and after treatments for LBP and NRP respectively, each patient being used as one's own control, thus each patient providing one pair of pre and post scores.

\section{Data Management}

The Excel spread sheet for data collection was designed to have these column headings besides the patient file number:a) age, b) gender, c) main health complaint (LBP), d) number of visits or treatments or doses, e) BaseRODI, f) PostRODI, g) RODIgain, h) BaseNDI, i) PostNDI, and j) NDIgain. There was also a 'Remarks' column to record any significant comment from patientsabout functionality and pain.

Nominal data on the electronic patient health record system (used for recording just basic data such as patient number, gender, age, date and time of visit, and other details) was gathered and this was cross-checked with data collected manually from clinic files. Treatment occurs in a continuum, in a cycle of appraisal, treatment and reappraisal. And so at what point should post hoc (postRODI) data be collected? For obvious reasons PostRODI and PostNDIdata were recorded on the last visit of the patient just before a cut-off date when this research effort commenced. The raw clinical data for 86 patients along with the embedded subset data for NDI was then posted to the IBM SPSS statistics software for processing.

\section{What Statistical Test to Apply?}

The following statistical tests were used to evaluate chiropractic effectiveness for LBP and NRP: paired samples t-test, repeated measures ANOVA, and Pearson linear correlation and multiple regression analysis. This somewhat immoderatetesting occurred even after learning that some of the demographics such as gender and age did not at all have a significant effect on the dependent variable, postRODI. Nor did the covariant factor of visits or doses affect the dependent variable significantly.But what if they are confounding variables interfering with the test results? It was later that we found that even in such a circumstance they are randomized or controlled by the nature of the pre-post measurement variables.

Secondly, the study was ad hoc and not preplanned. Non-parametric tests such as the Sign test and Wilcoxon tests were also used even after learning about the normality of the data purely to see what outcomes would come to light.Such tests came into play because of the initial use of the not-so-precisemeasurement variable of analog pain sensitivity index (PSI) data which was later discarded.

Third, underlyingthis maiden effort was an implicit intention to showcaseanalytical options available for future studies using data of PRO origin. Consequently different test statistics were attempted even if they overlapped such as paired t-tests and repeated measures ANOVA (rANOVA), and regression analysis too. Currently however, this study is keeping in mind the "Checklist: Guidance and key considerations for developing a statistical analysis section of an observational CER protocol". ${ }^{25]}$ In the light of these guidelines and comments received, reported below are just the details of the paired samples t-tests and rANOVA.

\section{Paired Samples T-Test}

The paired samples t-test spotssignificant differences if any between the means of the pretreatment data (BaseRODI and BaseNDI) and the means of post-treatment (PostRODI and PostNDI) data. Matched pairRODI as well as NDI data was gathered before and after treatment.NP related analytical tests for the $n=42$ subset were done separately.

Repeated measures analysis is suitable to a) minimize excess variability due to gender, age and other differences among patients, b) use even smaller samples to obtain accurate results, c) capture trends in effectiveness of treatment and d) not violate the assumption of independence under ANOVA. It can be useful later if and when a comparative effectiveness ranking is required to be made about methods such as Gonstead, Grostic, Thompson or any other technique. This is the reason that both the unidirectional paired samples t-test commonly used for pre and post data and the bidirectional repeated measure rANOVA were done. 
Correlation and regression exercises were undertaken to test the influence of age, visits, gender and baseRODI on the dependent variable RODIgain. The tests showed that none of the demographics had significant effectson the dependent variable BaseRODI.The predictor regression equation was:

PostRODI $=-9.212 \beta_{0}+0.174 \beta_{1}+(-) 0.041 \beta 2+0.589 \beta_{3}+2.94 \beta_{4}$

Where $\beta_{0}=$ the intercept, $\beta_{1}=$ age, $\beta_{2}=$ visits, $\beta_{3}=$ BaseRODI and $\beta_{4}=$ gender

$\mathrm{R}^{2}=0.49, \mathrm{p}$-value $=0.0000$

\section{Results}

Fig. 1: Histogram of RODIgain with bell normal curve overlay

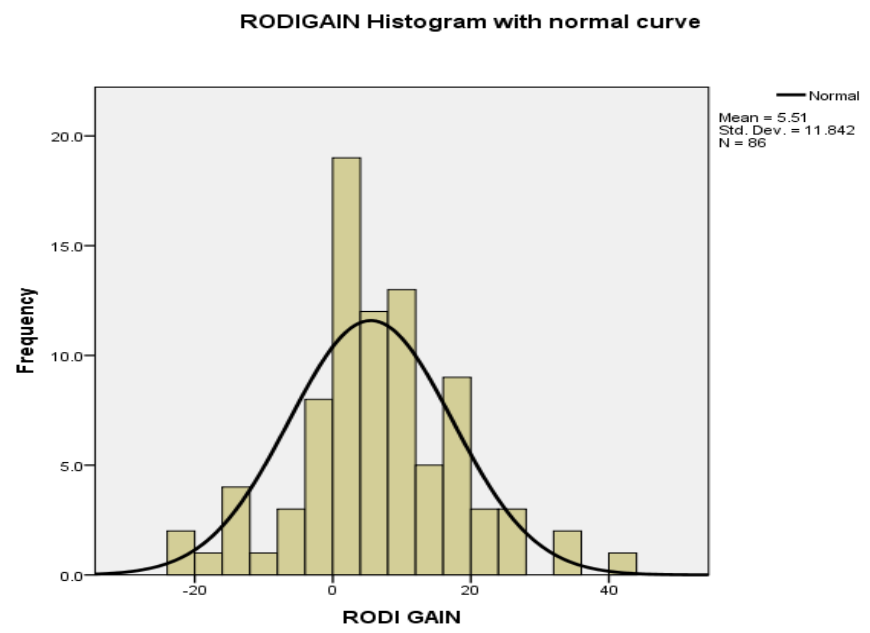

The histogram in Fig. 1 shows that RODI data distribution with an overlay of the bell normal curve. The confidence intervals would include thecentral value of a mean derived from the data. This would be representative of chiropractic clinics at $95 \%$ confidence level. Population parameters (including mean, variance and standard deviation) were reliably within the upper and lower bounds of the confidence intervals for each of the parameters particularly so when the data was trimmed for outliers.

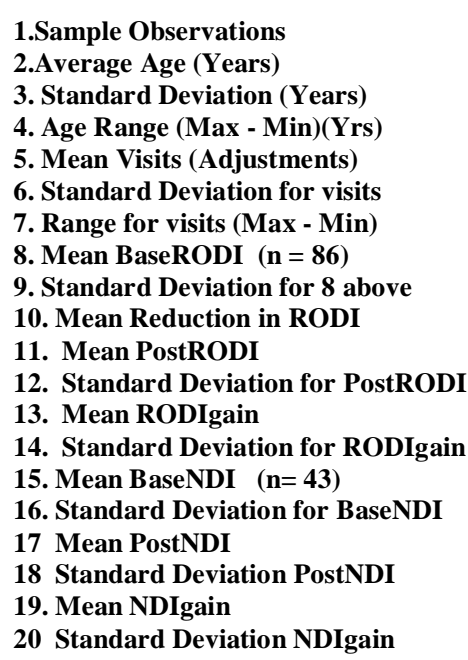

Table 1 Clinic Patient Profile

$\begin{array}{lll}\text { Male } & \text { Female } & \text { Total } \\ 43 & 43 & 86 \\ 51.6 & 50.2 & 50.9 \\ 18.2 & 20.6 & 19.3 \\ 62 & 64 & 66 \\ 5.9 & 5.3 & 5.6 \\ 3.6 & 2.6 & 3.2 \\ 22 & 9 & 22 \\ 21.7 & 24.3 & 23 \\ 15.85 & 14.43 & 15 \\ 5.3 & 5.2 & 5.73 \\ 15.49 & 19.49 & 17.27 \\ 14.71 & 14.51 & 14.53 \\ 5.26 & 5.21 & 9.55 \\ 10.9 & 12.54 & 13.26 \\ 9.53 & 9.81 & 19.7 \\ 12.6 & 14.21 & 12.67 \\ 7.58 & 6.33 & 13.64 \\ 10.57 & 10.54 & 11.16 \\ 1.95 & 3.49 & 2.64 \\ 6.87 & 6.03 & 6.44\end{array}$

RODI Is Revised Oswestry Disability Index, NDI Is Neck Disability Index. Computed by the authors.

RODI data indicates (Table 1) that male patients had marginally larger RODIgain from treatment though the mean baseRODI for females is larger at 24.3 against 21.7 for males. A partial explanation is that the average number of visits for males is more than for females. But conceivably there is more to this, and is dealt with under 'Discussion.' As the standard deviations would imply, the spread or variance in RODI gain is narrower for males and is more pronounced for females reflecting what was reported on the Oswestry form. While the mean baseline for NDI is 19.7 and the standard deviation 12.67, in the case of the reduction in NDI (NDIgain) the mean is 2.64 but the standard deviation is much larger at 6.44 , indicating the wide dispersal and variance. 
Table 2: Correlation BetweenKey Variables

\begin{tabular}{|l|c|c|c|}
\hline & $\begin{array}{c}\text { Correlation coef, } \\
\mathrm{r}\end{array}$ & Critical $\mathrm{r} \pm:$ & P-value (two-tailed): \\
\hline 1. Age and number of visits (treatments) & 0.0912797 & 0.212041 & 0.40324 \\
\hline 2. Age and BaseRODI & 0.1560692 & 0.212041 & 0.15130 \\
\hline 3. Number of visits and RODIgain & -0.0353898 & 0.212041 & 0.74632 \\
\hline 4. BaseRODI and RODIgain & 0.4137596 & 0.212041 & 0.00007 \\
\hline 5. BaseRODI and PostRODI & 0.6620382 & 0.212041 & 0.00000 \\
\hline 6. BaseRODI and baseNDI ( $\mathrm{n}=42)$ & 0.753 & 0.304395 & 0.00000 \\
\hline 7. BaseNDI and PostNDI $(\mathrm{n}=42)$ & 0.753 & 0.304395 & 0.00000 \\
\hline 8. BaseNDI and NDIgain & 0.514 & 0.304395 & 0.0005 \\
\hline
\end{tabular}

The main variables do not all correlate significantly with each other with the exception of BaseRODI and PostRODI, as well as BaseRODI and BaseNDI. BaseNDI and PostNDI correlate significantly as do BaseNDI and NDIgain, the last pair correlating at $r=0.5136$, critical value $\pm 0.3044, p$-value $=0.0005$. RODIgain and NDIgain correlate at $r=0.3689$, with critical $r$ at \pm 0.3044 and $p$-value at 0.01621 . Number of visits or treatments and NDIgain correlated negatively at $r=-0.1904$, $p$-value $=0.22716$. The same pattern obtains for age and NDIgain. Much of this data is presented in Table 2. As row 3 shows, two variables, namely, the number of treatments or visits and incremental reduction in RODI (RODIgain) correlate negatively. BaseRODI and postRODI are significantly correlated with $\quad r=0.66(r=0.702$ when $n=42)$ and a critical value of \pm 0.2108112 , $\mathrm{p}$-value $=0.0000$ ) and a predictive linear regression: postRODI $=2.99268+0.6426868$ BaseRODI

as shown in line 5 in Table 2 and in Fig. 2. BaseNDI and PostNDI correlate at 0.753, p-value $=0.000$.

Fig.2: PostRODI regressed on BaseRODI

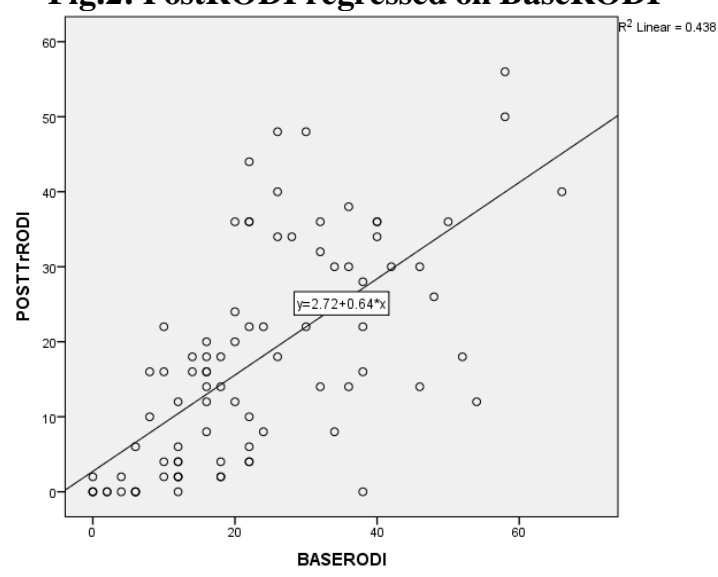

Fig. 3: Correlating Visits and RODIGAIN with interpolation line

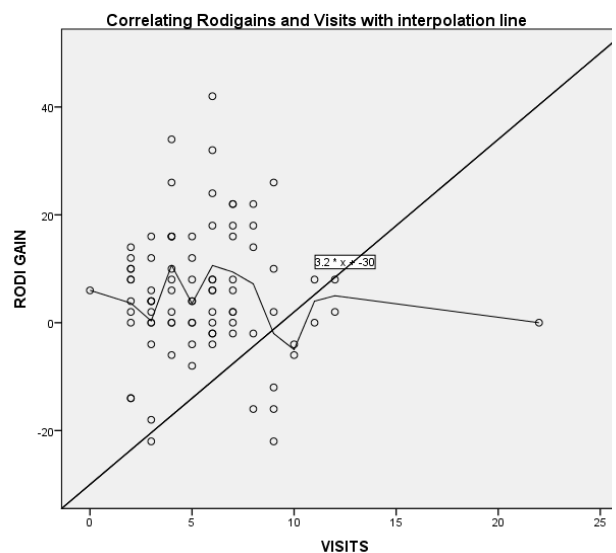

Fig. 2brings out the significant correlation between BaseRODI and PostRODI, the latter as a dependent. The same trends are substantiated in Tables 5 and 6 which presents identical data for BaseNDI and PostNDI.

In Fig. 3 the interpolation line is of interest. It trends down or remains flatafter the eighth treatment. The sample data does not provide enough evidence to support linear correlation between visits or doses and 
RODIgain or improvements in LBP even after an outlier observation of 22 doses was trimmed off from the sample. The slope is barely flat on the graph with almost an equal number of observations scattered above and below it. The slope is measured at -0.039 . The trimmed mean for visits is 5.4 with a standard deviation of 2.6 and the corresponding RODIgain mean is 5.58 with a standard deviation of 11.8. This association cannot be further sorted out, as will be explained under 'Discussion' because of dearth of observations after 12 doses, except for one visit number of 22 seen in Fig.3. There needs to be more observations in the upper ranges to be able to proceed further.

\section{Repeated Measures Anova}

Table 3: Analysis of Variance (ANOVA) of Before, After and Gain (RODI Data)

\begin{tabular}{|c|c|c|c|c|c|c|}
\hline Source & DF & SS & MS & Test Stat, F & Critical F & P-Value \\
\hline Treatment & 2 & 13557.754789 & 6778.877395 & 34.8296 & 3.030785 & 0.000 \\
\hline Error & 256 & 50214.482759 & 194.629778 & & & \\
\hline Total & 258 & 63772.237548 & & & & \\
\hline
\end{tabular}

Table 4: Univariate Analysis of Variance

Tests of Between-Subjects Effects

Dependent Variable: POSTRODI

\begin{tabular}{|c|c|c|c|c|c|c|c|}
\hline \multicolumn{3}{|l|}{ Source } & Type III Sum of Squares & $\mathrm{df}$ & Mean Square & F & Sig. \\
\hline \multirow{2}{*}{\multicolumn{2}{|c|}{ Intercept }} & Hypothesis & 45.827 & 1 & 45.827 & .563 & .467 \\
\hline & & Error & 1020.204 & 12.540 & $81.355^{\mathrm{a}}$ & & \\
\hline \multirow{2}{*}{\multicolumn{2}{|c|}{ AGE }} & Hypothesis & 143.995 & 1 & 143.995 & 1.774 & .208 \\
\hline & & Error & 974.005 & 12 & $81.167^{\mathrm{b}}$ & & \\
\hline \multirow{2}{*}{\multicolumn{2}{|c|}{ BASERODI }} & Hypothesis & 8677.118 & 28 & 309.897 & 3.858 & .000 \\
\hline & & Error & 2824.667 & 35.166 & $80.324^{\mathrm{c}}$ & & \\
\hline \multirow{2}{*}{\multicolumn{2}{|c|}{ VISITS }} & Hypothesis & 1089.861 & 12 & 90.822 & 1.130 & .366 \\
\hline & & Error & 3016.222 & 37.535 & $80.358^{\mathrm{d}}$ & & \\
\hline \multirow{2}{*}{$\begin{array}{l}\text { BASERODI } \\
\text { VISITS }\end{array}$} & $*$ & Hypothesis & 2568.980 & 32 & 80.281 & .989 & .538 \\
\hline & & Error & 974.005 & 12 & $81.167^{\mathrm{b}}$ & & \\
\hline
\end{tabular}

a. .019 MS(VISITS) +.000 MS(BASERODI * VISITS) +.980 MS(Error) b. MS(Error)

c. $.951 \mathrm{MS}($ BASERODI $*$ VISITS $)+.049 \mathrm{MS}($ Error $) \quad$ d. $.913 \mathrm{MS}($ BASERODI $*$ VISITS $)+.087 \mathrm{MS}($ Error)

The ANOVA F Test statistic is 34.8296 (Table 3) whereas the F Critical Value is 3.03 helping to reject the claim of equal mean values for pre and post RODI ( $\mathrm{p}$-value $=0.000$.)

Table 4 is a repeated measures between-subjects ANOVA table with the impact of different variables such as age, number of treatments and BaseRODI on the dependent variable, PostRODI. While the number of treatments and age do not seem to contribute much variance to the sample, it is BaseRODI that has a significant if not robust effect with the $\mathrm{F}$ test score at 3.858 ( $\mathrm{p}$-value $=0.000$.)

\section{PairedStudent T-Tests ForBaseRODI And BaseNDI}

Table 5: Paired Samples Statistics

\begin{tabular}{|l|c|c|c|c|c|}
\hline \hline \multicolumn{2}{|c|}{} & Mean & N & Std. Deviation & Std. Error Mean \\
\hline \multirow{2}{*}{ Pair 1 } & BSLRODI & 24.86 & 42 & 15.668 & 2.418 \\
\cline { 2 - 6 } & POSTRODI & 19.86 & 42 & 14.526 & 2.241 \\
\hline \multirow{2}{*}{ Pair 2 } & BSLNDI & 19.71 & 42 & 12.792 & 1.974 \\
\cline { 2 - 6 } & POSTNDI & 13.86 & 42 & 11.152 & 1.721 \\
\hline
\end{tabular}

Table 6: Paired Differences for BaseRODI and BaseNDI

\begin{tabular}{|c|c|c|c|c|c|c|c|c|c|}
\hline & & & & & & & $\mathrm{T}$ & df & $\begin{array}{l}\text { Sig. (2- } \\
\text { tailed) }\end{array}$ \\
\hline & & Mean & $\begin{array}{c}\text { Std. } \\
\text { Deviation }\end{array}$ & $\begin{array}{l}\text { Std. Error } \\
\text { Mean }\end{array}$ & $\begin{array}{l}95 \% \text { Confidence } \\
\text { Interval of the } \\
\text { Difference }\end{array}$ & & & & \\
\hline & & & & & Lower & Upper & & & \\
\hline Pair 1 & $\begin{array}{l}\text { BASERODI- } \\
\text { POSTRODI }\end{array}$ & 5 & 11.706 & 1.806 & 1.352 & 8.648 & 2.768 & 41 & 0.008 \\
\hline Pair 2 & $\begin{array}{l}\text { BASENDI- } \\
\text { POSTNDI }\end{array}$ & 5.857 & 8.547 & 1.319 & 3.194 & 8.521 & 4.441 & 41 & 0.000 \\
\hline
\end{tabular}

The paired tests for BaseRODI - PostRODI as well as BaseNDI - PostNDI $(n=42)$ brought out significant outcomes, student $t$-test data validating research findings of substantial amelioration of LBP (p-value $=0.008)$ and NRP ( $\mathrm{p}$-value $=0.000$ ) as shown in Table 6. When the same test is done with $\mathrm{n}=86$ larger sample, the null hypothesis of equality of means (BaseRODI = PostRODI, or no improvement in RODI) is rejected with the following test results: Test Statistic t: 14.1048 , Critical t: \pm 1.9883 , P-Value: 0.0000 . Again, as 
regardsimprovements in LBP or RODIgain $(n=86)$ itshowed there is evidence to reject null hypothesis of equality of sample means: Test statistic Student $\mathrm{t}: 4.3303$, critical $\mathrm{t}: \pm 1.9983$, and $\mathrm{p}$-value $=0.0000$. By all accounts this is statistically significant. In terms of the traditional approach of comparing the test statistic to the critical values as well asby means of comparing the p-value to the significance level $\alpha=0.05$, the null hypothesis of equal means (implying no change in baseline and post-treatment RODI) is rejected. There is evidence to support the alternative research hypothesis of unequal means or change for the better in terms of functionality.

Table 5 presents basic data for variables in the smaller subsample, $n=42$, such as BaseNDI and PostNDI with smaller standard error of the mean than for RODI means. NDI data thus appears to be more normally distributed than RODI data.

\section{Non-Parametric Techniques}

For the Sign test, the $\mathrm{Z}$ test statistic is -3.7 and the Critical $\mathrm{Z}$ is \pm 1.96 enabling the rejection of the claim that treatment does not make a difference. One of the learning moments was when a couple of faculty evinced interest and started using this method to determine summary efficiency of treatments for their own patients.

\section{Discussion}

The analyses of the chiropractic clinic post hoc data by means of different statistical tests bear out that the treatment for LBP at the clinic stands a good chance of success. This can be backed up by aggregate clinic patient data too. Out of a sample of 86 as many as 55 patients, or 64 percent got better, ( $p$-value $=0.0097$ ) three patients believed their LBP got worse, and 28 others reported no change in their status (noting a zero on the RODI form.) The mean reduction (or improvement) in RODI was 5.5 as against a mean baseRODI of 23. Out of a total of 42 patients 31 patients with NP felt better giving a success rate of 73.8 percent in terms of reduction in NDI (p-value $=0.0020$ ). Six patients felt worse after treatment and five patients reported no improvements (zero on the NDI.) The mean BaseNDI was 19.7 and the mean reduction (improvement) in NDI was 5.9. The mean PostNDI was 13.64. The difference between genders in both BaseRODI and PostRODI was not significant.

\section{Learning Moments in RODIand NDI Data Analysis Dosage or Number of Visits and BaseRODI}

Data analysis suggests a not significant associationor correlation between doses or number of visits to the clinic and reduction in RODI (postRODI.) This could be counter intuitive. At the same time it would be too early to draw a conclusion for several reasons. This would have to be recontextualized with reference to specific groups of patients, clinic, location and after adjusting for confounding factors if any. ${ }^{[26]}$ Before that it is crucial for any meaningful analysis to get more observations and enlarge the sample size. First in our sample there were not many patients that received more than 10 to 12 doses thereby offering no evidence of outcomes for doses larger than 10 or 12 .

Previous clinical trials such as by Haas et al do show "substantial linear effects with visits." In recent studies Haas et al concluded that "Overall, 12 visits yielded the most favorable results but was not well distinguished from other dose levels." ${ }^{[27,28]}$ The2004 study was with and without physical modalities (PM) such as hot tissue therapy and hot packs, thereby making it difficult to compare with our findings, if notconfounding the results.Our findings, based on post hoc empirical data, do not yet lead to the same conclusion of the presence of a probable declining marginal utility function in regard to doses or clinic visits. Several local factors do influence them such as physical condition of patient such as obesity or BMI, insurance coverage and economic status of patients, awareness of treatment availability and so forth. Most significantly a normal distribution of doses and more evidence about outcomes of larger doses could help avoid inaccurate conclusions.

Tests and results shown above are illustrations of learning moments. If a much larger sample with a more inclusive distribution of doses above 10 or 12 treatmentsshows that there is no significant correlation between number of treatments and the reduction in RODI that could help with validation or revision of current procedures. The fact of lack of association between doses and reduction in LBP (RODIgain) could take one by surprise unless the significant influence of BaseRODI level is examined coincidentally. This gives rise to questions how one could control for any unmeasured factors hidden from view and that could confound results. This is one good reasonwhy this study is notunambiguous in the matter of doses notwithstanding the negative sign against the $\beta_{2}$ coefficient in the regression equation (1) above.

To thequestion: what is the number of treatments needed to arrive at clinically acceptable RODI and NDI scores this study is unable to come up with a definitive response. While each treatment may increase total gain in RODI, it may not do so with marginal RODIgain or the incremental increase in improvement with every visit.Further investigations may show that depending upon the acuteness of the LBP, after an ideal number of visits or adjustments such as seven or eight, (or the optimal 12 as Haas et al suggest) the reductions in RODI may not be more than marginal or what is worse, there may be even a negative effect. This is an insightful 
“eureka!" moment, or a moment of "spikiness" a learner may feel ${ }^{[29]}$ that can be further corroborated by enlarging samples such as this, by more testing or even by undertaking independent clinical tests for LBP and NRP.

\section{Age and Dysfunction}

There are other learning opportunities. There is wide credence to the gerontology-basedbelief that aging patients have more back complaints and accordingly need more remedial services. The scatter plot for the two variables (age and number of clinic visits) is almost flat indicating no correlation. The RODI sample does not provide enough evidence of linear correlation with computed $r$ at 0.091 and the critical value at \pm 0.21 . (PValue $=0.40324)$. Similarly, data does not support the claim that older patients with LBP may have more dysfunctional RODI baseline scores before treatment. (Computed $\mathrm{r}=0.1560$, Critical $\mathrm{r} \pm 0.2120$, P-Value $=$ 0.1513 ) Further investigations are called for to explore the lack of a positive correlation between age and LBP remedial services in the context of the report that "Spinal pain is a significant musculoskeletal problem among older patients." ${ }^{[30]}$ Most analysts know that age not infrequently plays a confounder without a conventional or predictable behavior. In this study too it is somewhat of an inconstant variable. If other factors are conspiring to moderate the demand for chiropractic services at the clinic from where the data was obtained such as lack of awareness or lack of insurance coverage, they would have to be found out.

At the next higher level of analyses post hoc data can be organized to rank Gonstead, Grostic, Thompson or other techniques by relative effectiveness. The Cochrane rationalization principle can then be applied to discard relatively less effective techniques if any. Discarding established medical practice is common and is known to occur in all classes of medical practice. ${ }^{[31]}$ This study as ex ante planning material to organize future academic programs and plans. Firmer conclusions about adjustment techniques on the basis of outcomes would help reduction of variation in treatment and its upgrading.

\section{Circular Logic in Post-Hoc Data}

\section{Limitations}

There are views critical of post hoc studies like this. ${ }^{[32]}$ It is believed that they are not helpful because of circular logic. If a chiropractic researcher is looking for subluxation that is what he or she finds from post hoc data.One is "locked into a circular process." Plausibly,there could be 'circular logic' insome post hoc studies. In this study there was no premise to start with like 'looking for subluxation', nor was there any scrutiny of any reasoning underlying a health complaint. The criticism is therefore not germane to this study as there was no preconceived notion or 'confirmatory bias' to start with. PROs (patient reported outcomes) could serve as a hypothesis generating data source if patient data are routinely and accurately collected and compiled. ${ }^{[33]}$ As the study commenced the only objective was to gather post hoc data and outline an evidence report so as to learn more about the characteristics of key variables.After that the aim was to take the analysis to the next higher level to discover associations if any, amongst the key variables and gain new insights.Incidentally, the research effort also helped learn about the effectiveness of the treatment. The authors stayed neutral to the outcomes of the study throughout despite the stakes involved.

Second, the main problem with post-hoc data is it is not on par with randomized placebo-controlled clinical research data that is a priori well-planned. Nonetheless, if one could vouch for the authenticity of the post-hoc data, as the authors do, such data too would augment knowledge of chiropractic treatment, its remedial efficiency for LBP or NRP, optimal number of treatments, LBP incidence in connection with age and related matters. Significantly, neither the interns nor the doctors knew that clinical patient data was ever going to be used any which way, other than patient by patient evaluation, leaving little scope for data contamination.

\section{Sample Size}

Third the sample size of 86 in this study is adequate only for purposes of exploratory or pilot studies, but to be more persuasive it needs to be larger. The sample size was more influenced time constraints than by a desire for a holographic portrayal of the population.

Here again if it is the mean or a center of values that is being estimated, a smaller sample would do than when it is the proportionthat is to be estimated. A fragility of this study on account of the samples being not large enough is that the variances are pretty large. This does not however, detract from the significance of the exploratory study. Random variations get minimized when the sample sizes are "enormous." ${ }^{[34]}$ However, thesample size for estimating central values is adequate.

Four, the general perception of post hoc data is that it is somewhat contaminated bybias, slightly different from the one mentioned in 'Limitation' one above. First, while the superiority of facts from a priori planned clinical research is not questioned, it is iniquitous to assume away that all post hoc information is intrinsically flawed. With the introduction of mandatory electronic health records for every patient, the quality of patient data is improving. Where patient opinions on forms such as RODI are frank and candid, and where 
doctors and interns are self-persuaded by the professional advantages of objective patient data regarding treatment and its effects on a patient's LBP or any other complaint, it is not likely to be biased and could be as clean. Such data couldhelp draw valid statistical inferences, and especially for exploratory or pilot studies. The clinic authorities come to get a better grasp of the efficiencies or otherwise of the methods of treatment.

\section{Conclusions}

Post hoc data spawned by digital and manual documentation of patient diagnostics and treatment have been used conventionally in the evaluation of chiropractic interns who need to score at least the threshold percent score to avoid re-rotation in the clinic. The same data in aggregate form was used in this study to demonstrate varied other uses of post hoc data. It helped size up effectiveness of chiropractic treatment of LBP and NRP. The analytical study also yielded other hypothesis generating information that could a) help chiropractic decision-making and b) open further inquiries into some fuzzy areas such as if there is an effective optimal dose of treatments for every clinic and if the gain in RODI tapers off after an optimaldose.

\section{Funding and Conflicts Of Interest}

This study was funded internally. There were no conflicts of interest. The authors, as employees of a chiropractic university, undertook the study on their own to drawacademic attention to the advantages of a study of post hoc data in clinic patient files.

\section{Acknowledgment}

In the completion of this research work the authors consulted with a number of chiropractic clinic doctors, staff and student interns at the C-HOP Life University Clinic, and obtained clarifications of clinical terms besides valuable suggestions. They helped with dry runs in patient data location, collection, verification and organization. Dr. Kathryn Hoiriis, Director, Center for Excellence in Teaching and Learning gave much time going through the paper at various stages, editing and correcting errors. She helped put the text in the right sequence. Dr. Robert Rectenwald, Clinic Doctor, discussed some of the issues in this paper and facilitated the research. Dr. Stephanie Sullivan, Director, Office of Sponsored Research and Scholarly Activity (OSRSA), encouraged and propelled the research endeavor all through. Ms. Camille Sullivan, in Dr. Ralph Davis' office coordinated communications skillfully between the authors and expedited the formalities. The authors thank each one of these persons.

\section{References}

[1]. Flay BR, Biglan A, Boruch RF et al. Standards of Evidence: Criteria for Efficacy, Effectiveness and Dissemination. Prevention Science (C2005) DOI:10.1007/s11121-005-5553-y

[2]. Revicki DA, Frank L. Pharmacoeconomic evaluation in the real world. Effectiveness versus efficacy studies. Pharmacoeconomics 1999; 15: 423-434. | Article | PubMed | $\underline{\text { CAS } \mid}$

[3]. Fritz JM and Cleland J. Effectiveness Versus Efficacy: More Than a Debate Over Language (Editorial) Journal of Orthopaedic\& Sports Physical Therapy, 2003, Volume: 33 Issue: 4 Pages: 163-165 doi:10.2519/jospt.2003.33.4.163 http://www.jospt.org/doi/abs/10.2519/jospt.2003.33.4.163\#.U9GVt_ldXng Accessed July 15, 2014

[4]. Meade, T., Dyer. S., Browne, W., Townsend, J.,\& Frank, A.,(1990). Low back pain of mechanical origin: Randomized comparison of Chiropractic and hospital outpatient treatment. British Medical Journal 300, 1431-1437 Also see Meade et al (1995) Randomized comparion of chiropractic and hospital outpatient management for low back pain: Results from extended follow up. British Medical Journal, 311,349-351

[5]. Nyiendo J, Haas M, \& Goodwin P. Patient characteristics, practice activities and one-month outcomes for chronic, recurrent lowback pain treated by chiropractors and family medicine physicians: A practice based feasibility study. Journal of Manipulative and physiological Therapeutics, 23(4), 239-245

[6]. Burke J, Feasibility of using the Patient Reported Outcomes Measurement Information System (PROMIS) in academic health centers: case series design on pain reduction after chiropractic care, (2014) Platform presentation abstracts. Journal of Chiropractic Education: Spring 2014, Vol. 28, No. 1, pp. 55-82

[7]. Hinton PM, McLeod R, Broker B, MacLellan CE.Outcome measures and their everyday use in chiropractic practice. J Can Chiropr Assoc. Jun 2010; 54(2): 118-131. Available at http://www.ncbi.nlm.nih.gov/pmc/articles/PMC2875909/

[8]. Moses LE. The series of consecutive cases as a device for assessing outcomes of interventions. In: Bailar III J. and Mostellar F, editors. Medical Uses of Statistics. ${ }^{\text {nd }}$ Ed. Boston: 1992. Chapter 7, NEJM Books.

[9]. Lise Hestbaek L, Stochkendahl MJ. The evidence base for chiropractic treatment of musculoskeletal conditions in children and adolescents: the emperor's new suit? Chiropractic andOsteopathy,v.18;2010,PMC2891802.For online access: http://www.ncbi.nlm.nih.gov/pmc/articles/PMC2891802/?report=classic Accessed November 12, 2013

[10]. Lew M, Snow G. Radiographic utilization and demographics in a college teaching clinic, Journal of Chiropractic Medicine, December 2012, 11/4: 242/248

[11]. Arzt M, Floras JS, Logan AG, Kimoff RJ, Series F, Morrison D, Ferguson K, Belenkie I, Pfeifer M, J Fleetham J et al. Suppression of central sleep apnea by continuous positive airway pressure and transplant-free survival in heart failure; a post hoc analysis of the Canadian continuous positive airway pressure for patients with central sleep apnea and heart failure trial (CANPAP), Circulation June 11,$2007 ; 115: 3173-3183$

[12]. Garner MJ, Aker P, Balon J, Birmingham M, Moher D, Keenan D, and Monga P: Chiropractic care of musculoskeletal disorders in a unique population within Canadian community health centers, J Manipulative Physiol Ther 2007 (Mar);20(3):165-170. 
[13]. Dansk V, Gedin F: Patient-reported health outcomes before and after chiropractic care among patients with neck or back pain - A national Swedish study, Karolinska Institutet, Solnavägen 1, 17177 Solna, Sweden. Accessed on July 30 , 2014 from http://www.kiropraktorhogskolan.se/wp-content/uploads/2014/01/Viktor.D-Filip.G-Final-Master-thesis-130530.pdf

[14]. Walker BF, French SD, Grant W, Green S: A Cochrane review of combined chiropractic interventions for low back pain, Spine: 1 February 2011 - Volume 36 - Issue 3 - p 230-242 doi: 10.1097/BRS.0b013e318202ac73

[15]. National Center for Biotechnology Information (NCBI), List of articles at: http://www.ncbi.nlm.nih.gov/pmc/?term=chiropractic\%20treatment\%20of\%20low\%20back\%20pain\%20-\%20scholarly\%20papers

[16]. Agency for Health Research Quality (AHRQ) Review of Initiatives. Available at http://www.ahrq.gov/research/findings/evidencebasedeports/index.htmlwellhttp://search.ahrq.gov/search?q=Chiropractic+treatment+for+back+pain\&entqr=0\&output=xml_no_dtd \&proxystylesheet=ARCHIVE_Front_End\&client=ARCHIVE_Front_End\&site=ARCHIVE_AHRQ_GOV\&Submit.x=19\&Submit. $\mathrm{y}=12$ Accessed November 12,2013

[17]. Dingley C, Daugherty K, Derieg MK, et al. Improving patient safety through provider communication strategy enhancements (vols. 1-4) available at Rockville, MD: Agency for Healthcare Research and Quality; 2008.

[18]. Carreon LY, Glassman SD, Howard J. Fusion and nonsurgical treatment for symptomatic lumbar degenerative disease: a systematic review of Oswestry Disability Index and MOS Short Form-36 outcomes. Spine J. 2008;8(5):747-755.

[19]. Bishop PB, Quon JA, Fisher CG, Dvorak MF: The chiropractic hospital-based interventions research outcomes (CHIRO) study: a randomized controlled trial on the effectiveness of clinical practice guidelines in the medical and chiropractic management of patients with acute mechanical low back pain. Spine J. 2010;10(12):1055-1064

[20]. Haas M, Goldberg B, Aickin M, Ganger B, Attwood M. A practice-based study of patients with acute and chronic low back pain attending primary care and chiropractic physicians: two-week to 48-month follow-up. J Manipulative Physiol Ther 2004:27(3):1609.

[21]. Leonard DK, Tuttle WE, Pfefer M. Use of a new traction-flexion device in a patient with Low Back Pain: a case study, J Chiropr Humanities 2001:10:120-7 www:http://download.journals.elsevierhealth.com/pdfs/journals/1556-3499/PIIS1556349913601382.pdf Retrieved 09/12/2013

[22]. Australian Acute Musculoskeletal Pain Guidelines Group. Evidence-based management of Acute Musculoskeletal Pain, Brisbane, Australia. 2003. Australian Academic Press Pty Ltd. p.1

[23]. Kornegay C, Segal JB. Selection of data sources in developing a protocol for observational comparative effectiveness research: a user's guide. Valentgas P, Dreyer N, Nourjah P, Smith S, Torchia M, editors, 2013. Agency for Healthcare Research and Quality (AHRQ) Publication No. 12(13)-EHC099. Rockville, MD; 7-20.

[24]. Cooper R, McKey H. Chiropractic in the US: trends and issues, Milbank Quarterly, 2003 March; 81(1): 107-138.

[25]. Arbogast PG, VanderWeele TJ. Chapter 10.Considerations for statistical analysis. Developing an observational CER Protocol: a user's guide. Velentgas P, Dreyer NA.,et al Eds., Agency for Healthcare Research and Quality, Rockville, MD. p.142. Available at www.ahrq.gov. Retrieved 07/23/2014.

[26]. McNamee R. Education: Regression modelling and other methods to control confounding. Occup Environ Med 2005;62:500506 doi:10.1136/oem.2002.001115 Available at http://oem.bmj.com/content/62/7/500.full Retrieved 04/03/2014

[27]. Haas M, Groupp E.,Kraemer D., Dose-response for chiropractic care of chronic low back pain, Vol.4, Issue 5, pages 574-583, September-October, 2004

[28]. Haas M, Vavrek D, Peterson D, Polissar N, Neradilek MB. Dose-response and efficacy of spinal manipulation for care of chronic low back pain: a randomized controlled trial.Spine J. 2014 Jul 1;14(7):1106-16. doi: 10.1016/j.spinee.2013.07.468. Epub 2013 Oct 16

[29]. Baker Ryan SJd, Goldstein AB, Heffernan NT. Detecting the moment of learning, U.S. Department of Education program grant \#R305A070440. Available at http://www.columbia.edu/ rsb2162/BGH-v17.pdf. Accessed 03/03/2014

[30]. Dougherty PE, Hawk C. et al. The role of chiropractic care in older adults Chiropractic \& Manual Therapies 2012, 20:3 doi:10.1186/2045-709X-20-3

[31] Mayo Clinic Procedings August 2013; nn(n):1-9 n http://dx.doi.org/10.1016/j.mayocp.2013.05.012www.,ayoclinicproceedings.org, Mayo Foundation for Medical Education and Research, Elsevier Inc. Retrieved 07/07/2013.

[32] Vernon H. Nurturing basic science research in chiropractic, Dynamic Chiropractic - August 13, 1993, Vol. 32-3, Issue 17. Available at http://www.dynamicchiropractic.com/mpacms/dc/article.php?id=42477

[33]. Kornegay C, Segal JB. Selection of data sources in developing a protocol for observational comparative effectiveness research: a user's guide. Valentgas P, Dreyer N, Nourjah P, Smith S, Torchia M, editors, Agency for Healthcare Research and Quality, January 2013. AHRQ Publication No. 12(13)-EHC099. Rockville, MD: Agency for Healthcare Research and Quality; 7-20.

[34]. Motulsky H. Intuitive Biostatistics, New York: Oxford University Press; 2010, p.177 